\title{
Lattice-spring modeling of graphite accounting for pore size distribution
}

\author{
Craig N Morrison ${ }^{1, \mathrm{a}}$, Andrey P Jivkov ${ }^{1, \mathrm{~b}}$, Gillian Smith ${ }^{2, \mathrm{c}}$ and John R Yates ${ }^{1, \mathrm{~d}}$ \\ ${ }^{1}$ School of MACE, The University of Manchester, Oxford Road, Manchester M13 9PL, UK \\ ${ }^{2}$ Interface Analysis Centre, University of Bristol, Tyndall Avenue, Bristol BS8 1TL, UK \\ accraig.morrison-2@postgrad.manchester.ac.uk, bandrey.jivkov@manchester.ac.uk, \\ `gillian.smith@bristol.ac.uk, djohn.r.yates@manchester.ac.uk
}

Keywords: Nuclear graphite; Porosity; Meso-scale deformation; Micro-cracks; Macroscopic behaviour

\begin{abstract}
Lattice models allow length scale dependent micro-structural features and damage mechanisms to be incorporated into analyses of mechanical behaviour. They are particularly suitable for modelling the fracture of nuclear graphite, where porosity generates local failures upon mechanical and thermal loading. Our recent 3D site-bond model is extended here by representing bonds with spring groups. Experimentally measured distributions of pore sizes in graphite are used to generate models with pores assigned to the bonds. Microscopic damage is represented by failure of normal and shear springs with different criteria based on force and pore size. Macroscopic damage is analysed for several loading cases. It is shown that, apart from uniaxial loading, the development of micro-failures yields damage-induced anisotropy in the material. This needs to be accounted for in constitutive laws for graphite behaviour in FEA of cracked reactor structures.
\end{abstract}

\section{Introduction}

Nuclear-grade graphite has featured in over 100 reactors [1] with its main functions being a fast neutron moderator and structural material. It also forms an integral part of a potential Generation IV Very High Temperature Reactor (VHTR). The integrity of graphite, as with all structural reactor components, is critical for their fitness for purpose. Understanding graphite's fracture behaviour is essential for approving plant life extensions and predicting in-service performance.

Synthetic graphite is manufactured from petroleum cokes and a binder material; usually coal-tar pitch. The resulting micro-structural features - grain size, pore size/density, are strongly influenced by the manufacturing process and the structure of the coke and binder particles used. The work in this paper focuses on Gilsocarbon, a relatively fine-grained graphite, used in the UK Advanced Gascooled Reactors (AGRs). Graphite microstructure consists of 3 phases; a matrix of graphitized binder particles, relatively large filler particles (derived from coke) and porosity [2]. In Gilsocarbon the spherical filler particles, ranging from $0.3-1.5 \mathrm{~mm}$ in size, result in near isotropic mechanical properties [3]. The 3 main porosity populations [4], ranging from $\mathrm{nm}$ to $\mathrm{mm}$ in size, total approximately $20 \%$ of virgin graphite volume. Gas evolution cracking occurs within the matrix as gas bubbles form when liquid pitch, impregnated to increase density, boils during baking [3]. Calcination and Mrozowski cracks form within filler particles due to uneven thermal expansion and shrinkage as the graphite heats and cools during calcination and graphitization respectively [2,5].

Graphite, alongside rock, concrete and cement, is considered a quasi-brittle material [6], with failure occurring when distributed micro-cracks coalesce into a critically sized flaw. As a result, graphite exhibits a reduced stiffness upon loading (similar to plasticity) prior to failure [2] despite remaining brittle. Using local approaches to model graphite failure can capture the dependence of macro-scale integrity on meso-scale features by incorporating mechanistic understanding of failure at the length scale of features [7]. However current methods rely on weakest link (WL) assumptions and fail to account for the micro-crack interactions apparent in quasi-brittle materials [8]. Lattice models are a branch of discrete, local approach models, consisting of nodes connected into a lattice through discrete elements including springs [9] and beams [10]. Element properties allow a material response according to actual mechanistic failure data. Unlike WL methods, lattice models are based around a parallel statistical system, with load redistribution amongst remaining bonds once a bond 
is broken. Such models have been developed for graphite [4,10,11] and cement [12]. The work presented here furthers the site-bond lattice model developed by Jivkov and Yates [13]. The model has been calibrated using a generalized continuum theory, with bond stiffness constants evaluated through strain energy equivalence of a discrete unit cell and classical continuum [14].

\section{Theory and model}

The site-bond model is based upon a tessellation of space into truncated octahedral cells, Fig 1a. Particles, attached to cell centres, interact via deformable bonds, with 14 bonds connecting a particle to its neighbours, Fig 1b. The 6 bonds in the principal directions and 8 bonds in the octahedral directions can have different mechanical responses. The energetic calibration for this model [14] suggested that as a first assumption particle rotations could be ignored, with bonds represented only by normal and shear springs. Spring elastic constants $K_{\mathrm{ax}}{ }^{\text {princ }}, \mathrm{K}_{\mathrm{ax}}{ }^{\text {oct }}, \mathrm{K}_{\mathrm{sh}}{ }^{\text {princ }}$ and $\mathrm{K}_{\mathrm{sh}}{ }^{\text {oct }}$ were calibrated for plane stress and plane strain for a graphite of typical properties $E=11 \mathrm{Gpa}$, $v=0.2[15]$ with values $4.041 \times 10^{4}, 4.939 \times 10^{4}, 2.245 \times 10^{3}$ and $4.49 \times 10^{3}$ respectively.

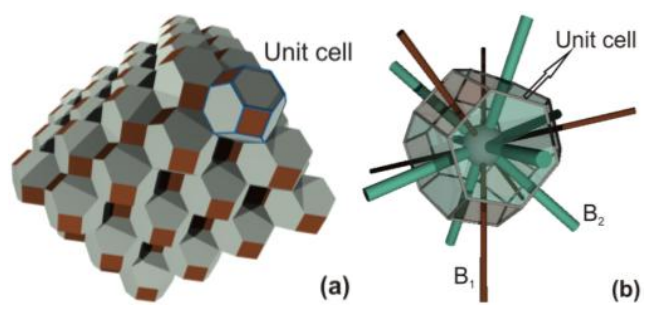

Fig 1. Cellular representation of material (a); and unit cell with bonds (b).

The behaviour of shear springs is illustrated in Fig 2a, where the shaded area represents energy dissipated in spring failure. For axial springs, failure in compression is not permitted and the behaviour is shown in Fig $2 b$.
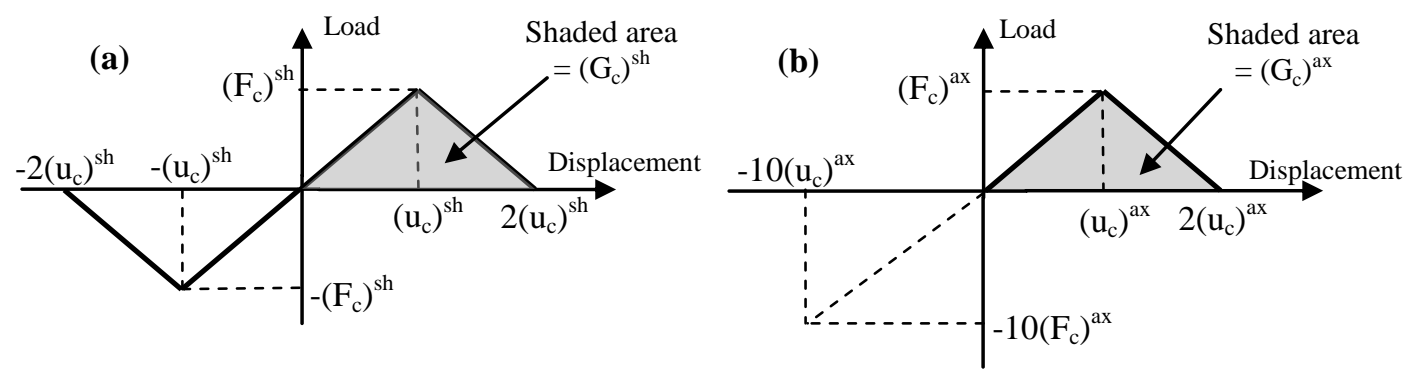

Fig 2. Spring failure criteria for shear springs(a); and normal springs (b).

Gilsocarbon microstructure was modeled, assuming porosity occurs exclusively within the matrix phase, with calcination and Mrozowski cracks ignored. Filler particles with normally distributed sizes were randomly located with total volume fraction of $20 \%$. Pores with normally distributed sizes were located in the matrix with a constraint preventing coalescence and $5 \%$ volume fraction. This porosity was distributed to the faces of the cellular structure. The ratio between pore and face areas determined the failure energy of the corresponding springs, so that $\mathrm{G}_{\mathrm{c}}$ varied between zero for very large pores and one for very small pores. The model was subject to uniaxial tension; $u_{1}=u, u_{2}=u_{3}=0$, as well as to fields experienced ahead a crack: high-constraint plane strain $\left(\mathrm{u}_{1}=\right.$ $\left.u_{2}=u, u_{3}=0\right)$ and lower-constraint plane strain $\left(u_{1}=0.5 u_{2}=u, u_{3}=0\right)$. For uniaxial tension the damage can be characterized by the parameter $D_{E}$, Eq. (1), as principal stresses $\sigma_{2}=\sigma_{3}=0$.

$$
D_{E}=1-\frac{\left(\sigma_{1} / \varepsilon_{1}\right)_{i}}{\left(\sigma_{1} / \varepsilon_{1}\right)_{0}}=1-\frac{E_{i}}{E_{0}} .
$$

For the plane strain cases, 4 damage parameters are required, Eq. (4-5), evaluated from a decomposition of nominal stress/strain into deviatoric and volumetric components of $s_{\alpha}, e_{\alpha}$ and $\sigma_{h}$, $\varepsilon_{v}$ respectively, Eq. (2-3), where $\sigma_{h}$ is hydrostatic stress and $\varepsilon_{v}$ is volumetric strain. 
$s_{\alpha}=\sigma_{\alpha}-\sigma_{h}$.

$e_{\alpha}=\varepsilon_{\alpha}-\varepsilon_{v} \quad$ where $\alpha=1,2,3$.

$D_{K}=1-\frac{\left(\sigma_{h} / \varepsilon_{v}\right)_{i}}{\left(\sigma_{h} / \varepsilon_{v}\right)_{0}}=1-\frac{K_{i}}{K_{0}}$.

$D_{\mu}=1-\frac{\left(s_{\alpha} / e_{\alpha}\right)_{i}}{\left(s_{\alpha} / e_{\alpha}\right)_{0}}=1-\frac{\mu_{i}}{\mu_{0}}$

Where $K$ and $\mu$ are the bulk and shear moduli respectively.

\section{Results}

Uniaxial tension exhibits quasi-brittle behaviour with softening before failure as expected, Fig 3 . The similarity of damage parameters, $D_{E}$ and $D_{\mu}$ suggests that the material remains isotropic, Fig 4.

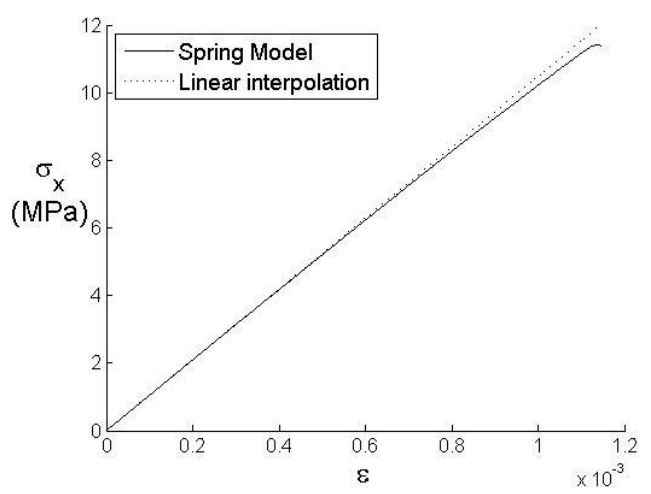

Fig 3. The stress/strain response of the model under uniaxial tension.
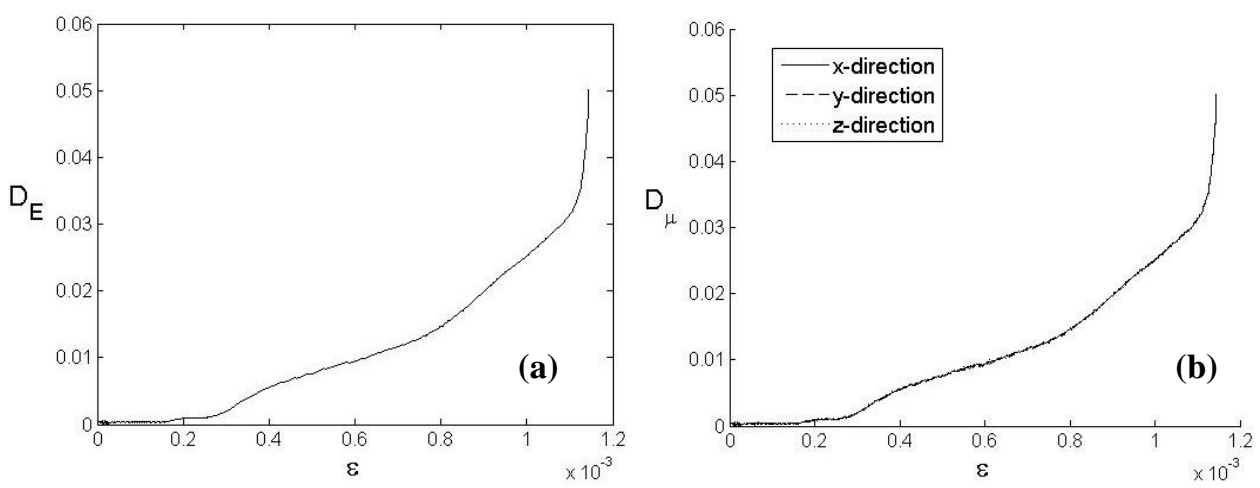

Fig 4. The damage parameters for uniaxial tension, $\mathrm{D}_{\mathrm{E}}(\mathrm{a}) ; \mathrm{D}_{\mu}(\mathrm{b})$.

Upon loading in plane strain under both high and low constraint the damage parameter $D_{\mu}$ becomes dependent on direction suggesting anisotropy is introduced, Fig $5 \mathrm{~b}$ and Fig $6 \mathrm{~b}$.
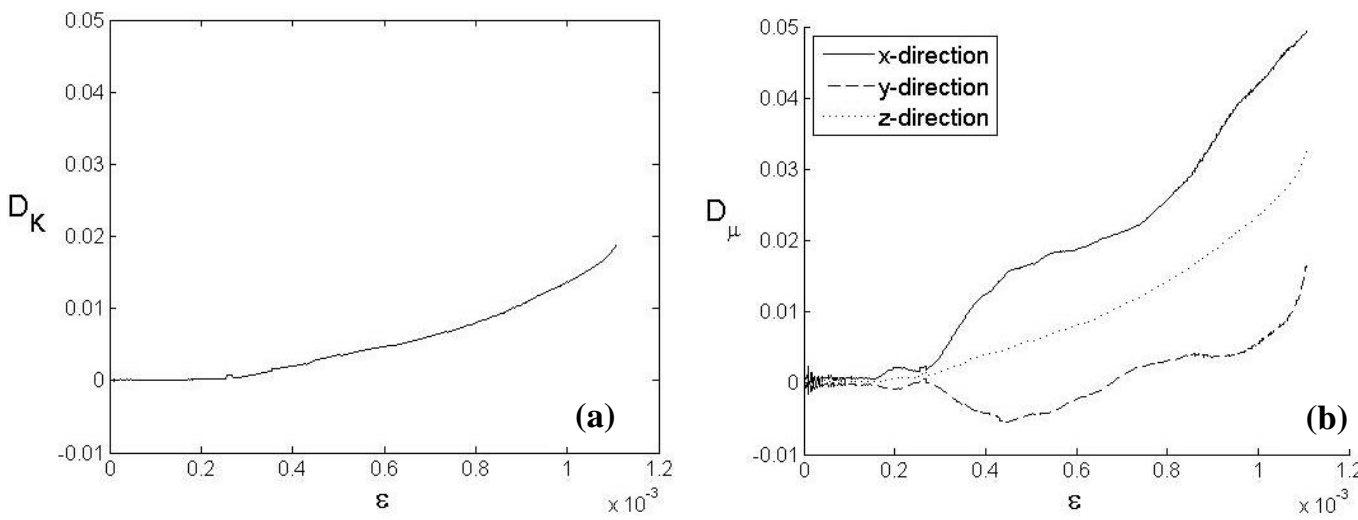

Fig 5. The damage parameters for plane strain high constraint $\mathrm{D}_{\mathrm{K}}(\mathrm{a}) ; \mathrm{D}_{\mu}(\mathrm{b})$. 

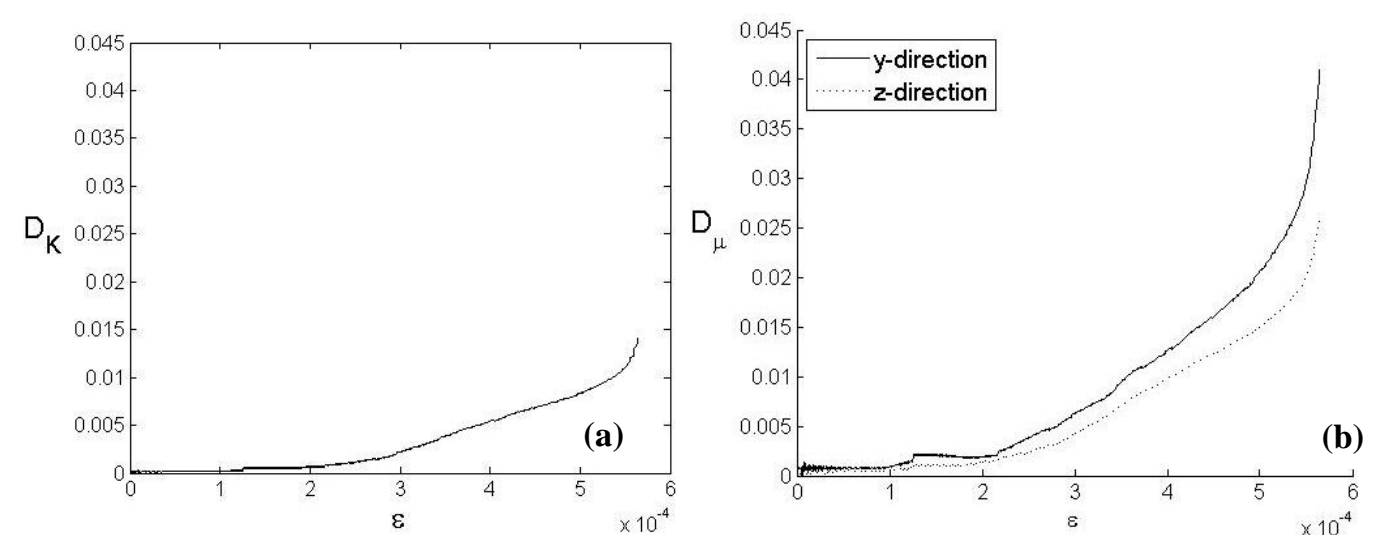

Fig 6. The damage parameters for plane strain low constraint, $\mathrm{D}_{\mathrm{K}}(\mathrm{a}) ; \mathrm{D}_{\mu}(\mathrm{b})$.

\section{Discussion and conclusions}

The results show that the development of micro-damage in porous graphite is strongly dependent on the loading. Uniaxial loading seems to be the only case where the material remains isotropic with damage evolution. This suggests that a macroscopic damage evolution law based on a single damage parameter is appropriate only for uniaxial states. For material ahead of a macroscopic crack, high-constraint, Fig 5, and low constraint, Fig 6, damage evolves differently in different loading directions. Negative damage parameter observed in Fig 5, is not surprising; it merely shows that the transverse shear modulus in $y$-direction increased above the initial isotropic value as the transverse shear modulus in $x$-direction decreased. It is also clear that damage of shear moduli is larger than the damage of bulk modulus. The results suggest that damage evolution laws for complex loading cases need to be based on three independent invariant of the stress tensor. Presently, it is not clear whether a load-independent evolution law can be developed for materials with given pore space structures with the strategy presented here. This is a subject of ongoing work.

\section{References}

[1] IAEA report (2006).

[2] A. Hodgkins, T. J. Marrow, M. R. Wootton, R. Moskovic, and P. E. J. Flewitt: Mater. Sci. Tech. Vol. 26 (2010), p. 899.

[3] M. R. Joyce, T. J. Marrow, P. Mummery, and B. J. Marsden: Eng. Fract. Mech. Vol. 75 (2008), p. 3633.

[4] M. R. Bradford and A. G. Steer: J. Nucl. Mater. Vol. 381 (2008), p. 137.

[5] E. Schlangen, P. E. J. Flewitt, G. E. Smith, a. G. Crocker, and A. Hodgkins: Key Eng. Mater. Vol. 452-453 (2010), p. 729.

[6] M. Mostafavi and T. J. Marrow: Fatigue Fract. Eng. Mater. Struct. Vol. 35 (2012), p. 695.

[7] J. Lemaitre: Eng. Fract. Mech. Vol. 23 (1986), p. 523.

[8] Z. P. Bažant and S.D. Pang: J. Mech. Phys. Solids Vol. 55 (2007), p. 91.

[9] A. Pazdniakou and P. M. Adler: Tran. Porous Med. Vol. 93 (2012), p. 243.

[10] E. Schlangen and E. Garboczi: Eng. Fract. Mech. Vol. 57 (1997), p. 319.

[11] N. N. Nemeth and R. L. Bratton: Nucl. Eng. Design Vol. 240 (2010), p. 1.

[12] P. Grassl, D. Grégoire, L. Rojas Solano, and G. Pijaudier-Cabot: Int. J. Solids Struct. Vol. 49 (2012), p. 1818.

[13] A.P. Jivkov and J.R. Yates: Int. J. Solids Struct. Vol. 49 (2012), p. 3089.

[14] C. N. Morrison, A. P. Jivkov, and J. R. Yates: Proc. ICF13 (Beijing, China, 2013), p. S31016.

[15] M. Holt: Issues of scale in nuclear graphite components (PhD Thesis, University of Hull, 2008). 